\title{
EFFECT OF LOW-LEVEL LASER THERAPY ON ORTHODONTIC INDUCED INFLAMATORY ROOT RESORPTION IN RATS
}

\author{
Ahmed M. Yassin ${ }^{1 *} B D S$, Fathy I. Shehata ${ }^{2} P h D$, Afaf A. Al-Sawa ${ }^{2} P h D$, Sahar S. karam² $P h D$
}

\begin{abstract}
INTRODUCTION: Low-level laser therapy (LLLT) has been studied in many fields of dentistry due to its reparative and anti-inflammatory effect. Regarding the orthodontic tooth movement, LLLT accelerates tooth movement, reduces the pain, enhances bone remodeling and reduces the orthodontic inflammatory induced root resorption (OIIRR).

OBJECTIVES: The aim of the present study was to assess histologically and histomorphometrically the effect of LLLT on OIIRR under heavy orthodontic force magnitude (50 g) in rat model.

MATERIALS AND METHODS: Sixteen male Albino rats weighing from (250-300 g) were randomly assigned to two equal groups (8 rats each). Control heavy-force group (CH): the maxillary right first molars were subjected to orthodontic force of 50 g by placement of a coil spring. Laser high-force group (LH): the maxillary right first molars were subjected to orthodontic force of $50 \mathrm{~g}+\mathrm{LLLT}$ at four points (two buccal and two palatal), 12 seconds per point, with a GaAlAs diode laser source irradiated with the dose of $4.8 \mathrm{~J} / \mathrm{cm} 2$ (100 mW, $12 \mathrm{~s}, 0.6 \mathrm{~J}$ ) on every other day during force application. The rats were sacrificed after 21 days of orthodontic force application. After sacrificing the rats; the maxillae were hemisected and serial sections of the area of maxillary right first molar were obtained and evaluated histologically and histomorphometrically.

RESULTS: Root resorption was significantly decreased $(\mathrm{p}=0.001)$ in laser group $(\mathrm{L})$ in comparison to the control group $(\mathrm{C})$. In contrast, root resorption was the largest and deepest up to the dentin in the control group. Moreover, odontoclasts were observed especially in the $\mathrm{C}$ group indicating still active root resorption activities. In addition, increased blood vasculature and formation of reparative cementum layer covering the resorption lacunae were observed in the Laser group.

CONCLUSIONS: LLLT seems promising in preventing or reducing orthodontic root resorption, in addition to accelerating and enhancing the healing and repair of OIIRR.

KEYWORDS: Orthodontics, root resorption, Low-level laser therapy, phototherapy, orthodontic force magnitudes.
\end{abstract}

1- Demonstrator of oral biology Faculty of dentistry, Alexandria University.

2- Professor of oral biology Faculty of Dentistry, Alexandria University.

* Corresponding author:

E-mail: dr.ahmedmagdy0099@gmail.com

\section{INTRODUCTION}

Orthodontic tooth movement is a process which includes application of external forces resulting in physiologic responses. The orthodontic force results in a minor reversible-injury to the tooth-supporting tissues, and subsequently the tooth movement occurs after physiologic adaptation of alveolar bone in response to this mechanical strain (1). The net result of remodeling changes that occur in the dental and paradental tissues including the dental pulp, periodontal ligament (PDL), alveolar bone and the gingiva leads to orthodontic tooth movement (2).

Root resorption of permanent teeth is a pathological process that results in the damage of mineralized dental tissues following mechanical or chemical injury to periodontal tissue and root surface $(3,4)$. Orthodontic induced inflammatory root resorption (OIIRR) is considered as a side effect of the cellular activity associated with the hyaline zone elimination process in an overcompressed periodontal ligament $(3,5)$. Compression force on periodontal tissues during orthodontic treatment results in ischemic necrosis, death of periodontal ligament cells, removal of the organic cementoid layer and exposure of root surfaces to the action of phagocytic cells and osteoclasts (3, 4). Root resorption starts as a consequence of the process of elimination of the formed hyaline sterile necrosis regions by the recruited inflammatory cells under the influence of local inflammatory mediators $(3,4)$.
Literature reveals the prevalence of OIIRR occurring during orthodontic treatment to be $3 \%-100 \%$. Several histologic studies reported that more than $90 \%$ of orthodontically treated teeth show OIIRR, while lower percentages were reported by studies based on diagnostic radiographic techniques (6). Regarding the degree of severity and according to Brezniak and Wasserstein (3); OIIRR can be classified to cemental or surface resorption, dentinal resorption, and circumferential apical root resorption (whole mineralized tissue components of the root apex are resorbed). The degree of OIIRR is usually considered mild, and does not compromise dental function in many cases, except for few (0.5\%) patients who have severe lesions damaging more than one-third of the root length (4).

Many etiological factors have been suggested to influence the root resorption process. These factors can be classified to factors related to patient biology or others related to treatment mechanics (7). Several biological factors reported in the literature include genetic factors (2, 8 ), age (2, 8-10), morphological characteristics of the root $(2,8-10)$, degree of density of bone $(2,8)$ and a previous history of external root resorption $(2,3,8)$. On the other hand, factors related to treatment mechanics that have been suggested to influence the OIIRR are magnitude of force (2, $8,11)$, treatment duration $(2,8-10)$, the distance of tooth 
movement $(2,8,9)$, tooth movement direction $(2,8)$, type of force application (intermittent versus continuous) $(2,3,8)$, type of appliance (3) and technique of the treatment $(2,8)$.

In order to minimize and/or repair the OIIRR; several chemicals and methods have been tested on human and animal study models. Some of these chemicals are fluoride (12), steroidal and nonsteroidal drugs $(4,13)$, calcitonin (14), human growth hormone (15), clodronate (16), Icariin (17), and tetracycline $(5,18)$. Other non-invasive and clinically suitable potential methods to prevent and repair the OIIRR are low-intensity pulsed ultrasound (LIPUS) (19) and low-level laser therapy $(\operatorname{LLLT})(1,4,5)$.

Among most of the other treatment modalities, and in contrast to the injected chemicals and orally-consumed medicaments, lasers do not cause any systemic side effects, discomfort nor affect the general health status of the patients (20). The effects of laser irradiation on the treated tissues range from biostimulation to micro-explosion (21). When the laser irradiation affects the tissue without causing local increase in the tissue temperature more than $1^{\circ} \mathrm{C}$, it is called 'biostimulation' (21). Treatments depend on using biostimulation power of laser radiation are called 'low-level laser therapy’ (LLLT) or laser acupuncture $(21,22)$.

At the cellular level, the absorption of wavelengths of red and infrared laser light increases the mitochondrial activities and cellular respiration resulting in increase of cellular energy and elevation of adenosine triphosphate (ATP) synthesis (22). Furthermore, the LLLT may affect RNA and DNA synthesis and so affect the cell proliferation, cellular activities and release of the growth factors (22). The action of LLLT on cell energy metabolism results in the activation of intracellular signaling cascades which reduce the expression of inflammatory cytokines and proinflammatory molecules, thus decreasing the inflammatory cell infiltration and activation, promotes the clearance of tissue debris, enhances cell differentiation and proliferation, induces angiogenesis, and accelerates the production of collagen and other matrix components (4).

LLLT promotes osteoblast proliferation and differentiation and consequently enhances osteogenesis (22). On the other hand, the effects of phototherapy on osteoclasts are still unclear. Literature reported the positive role of laser biomodulation on the osteoclast differentiation and activities through the increased c-fms gene expression and RANK/RANKL (22). However, LLLT inhibits osteoclast differentiation indirectly by upregulating the osteoprotegrin (OPG) production by the osteoblasts which has a negative effect on the osteoclast differentiation (22).

Moreover, LLLT positively accelerates wound healing and it is found to have an anti-inflammatory effect (4). As orthodontic tooth movement (OTM) includes controlled inflammatory process, many clinicians have tested the effect of LLLT on pain control (1), tooth movement velocity (21), bone remodeling (1) and root resorption during orthodontic tooth movement $(1,4,5,23)$.

Due to the reparative and anti-inflammatory effects of LLLT, it has been suggested that it could have a role in preventing, reducing or even repairing root resorption and improving periodontal healing $(1,4,5,24)$. However, few studies disagreed with that effect of the LLLT and claimed that there is no effect of LLLT on OIIRR (23). These conflicting results could be due to different laser application protocols and different operating parameters such as wavelengths, fluences, or laser powers.
Therefore, this study was conducted to assess the effect of LLLT on orthodontic inflammatory root resorption using heavy orthodontic force in a rat model.

\section{MATERIALS AND METHODS}

This research was conducted in accordance with the ethical principles of animal experimentation by the Institutional Ethical Committee of Faculty of Dentistry Alexandria University. The animal section was carried out in the animal house of the Faculty of Agriculture, Alexandria University. The histological section of the study was done in the Histological Unit of the Department of Pathology of the Medical Technology Center, a branch of Alexandria University Medical Research Institute at Smouha District in Alexandria.

Sixteen male Albino rats weighing from (250-300 g) were used for this experiment. The animals were kept in collective cages, four per cage, under normal laboratory conditions of temperature $\left(22-25^{\circ} \mathrm{C}\right)$, good ventilation and standard light/dark cycle (12/12 h) with free access to soft standard food and water ad libitum. The standard diet regimen had been replenished daily throughout the experimental period.

The 16 rats $(n=16)$ were randomly divided into two groups (each of 8 rats) as follows:

Heavy-force Control group (C); with (50 g) applied force $(n=8)$.

Heavy-force Laser group (L); with (50g) applied force $+\operatorname{LLLT}(\mathrm{n}=8)$.

\section{Induction of tooth movement}

Animals were placed under general anesthesia with intramuscular injection of xylazine $(30 \mathrm{mg} / \mathrm{kg})$ and ketamine (70 $\mathrm{mg} / \mathrm{kg}$ ) prior to orthodontic appliance placement. Each animal was immobilized with open mouth on a table specifically designed for this experiment.

A custom-made super-elastic closed coil spring (length, $10 \mathrm{~mm}$; eyelet diameter, $1.5 \mathrm{~mm}$ ) (DB Orthodontic Company, United Kingdom) was placed between the maxillary first molar and maxillary incisors (23). The coil was fixed to the maxillary molar and incisors using stainless steel ligature wire (0.008”) (DB Orthodontic Company, United Kingdom). The force magnitude was adjusted to be $50 \mathrm{~g}$ by the help of a force gauge (YDM Corporation, JAPAN). This force was chosen based on previous studies that showed that $50 \mathrm{~g}$ force on rat's molar induces tooth movement and root resorption (24). The teeth were covered by photo-cured flowable resin around the ligature wire to improve the appliance retention.

\section{Laser irradiation}

During the tooth movement, the experimental LLLT group (L) received laser irradiation by the following protocol:

The laser irradiation was performed using a GaAlAs diode laser (Simpler Doctor smile dental Diode laser, ITALY) $(\lambda=980 \mathrm{~nm}, 100 \mathrm{~mW}$ output power $)$ on the side of appliance.

The buccal and palatal sides of maxillary first molar were irradiated from four points (two points at buccal, two at the palatal side) for 12 seconds for each point. The irradiation was performed with continuous waves by a fiber applicator $3 \mathrm{~mm}$ in diameter every other day until the end of the experiment. Energy per point of application was 0.6 $\mathrm{J}$ with a total radiant energy of $2.4 \mathrm{j}$ for the net of the four points. Beam spot size at target equal $0.0314 \mathrm{~cm} 2$ with a total area of irradiation equal $0.125 \mathrm{~cm} 2$ (5). 
The rats were euthanized under anesthesia at the end of the experimental period (day 21) for both Laser and control groups.

\section{Tissue preparation for light microscopic examination and histomorphometry}

The maxillae were hemisected and cleaned of soft tissues, fixed in $10 \%$ neutral buffered formalin, decalcified, dehydrated in ascending concentrations of ethyl alcohol, cleared with xylene then embedded in paraffin.

4- $\mu \mathrm{m}$ Serial sections of maxillary right first molar, at the side of fixed appliance, were obtained parallel to the occlusal plane up to the radicular division using rotary microtome. Sections were stained with Hematoxylin \& Eosin stain for general examination and for morphometric analysis (25). A histological evaluation was done using a binocular microscope (OPTIKA microscope, Italy) with a 10x magnification ocular lens. The sections were photographed by using a digital camera (Nikon, Japan) connected to a computer.

The cross sections of the distal-buccal root of the right maxillary first molar were used for observation and quantification. For every specimen, all the sections, taken from the cervical part of the root to the root apex, were examined under light microscope for histological analysis, and five cuts, with the most expressive tissue events and best material quality, from the apical two thirds of the distalbuccal root were chosen for the histomorphometric analysis.

The software ImageJ (ImageJ, National Institute of Health, USA) was used to measure the amount of root resorption lacunae for histomorphometric analysis (26). The analyzed area corresponded to the disto-buccal root. In order to determine the area of resorption and its ratio to the total area of the radicular hard structures, the total root area was measured, considering an external virtual line that limited the resorbed foci and continued the root contour, then the area of the pulp cavity was subtracted from the total root area to determine the area of the hard dental structures of the root without the pulp. After that the resorbed areas were measured, considering the same external virtual line that limited the resorbed foci and continued the root contour, and the ratio of root resorption to the total root area was obtained.

\section{STATISTICAL ANALYSIS}

Data were collected and entered to the computer using SPSS (Statistical Package for Social Science) program for statistical analysis (version 21) (27). Kolmogorov-Smirnov test of normality revealed no significance in the distribution of the variables, so the parametric statistics was adopted (28). Comparisons were carried out between two studied independent normally distributed subgroups using independent sample t test (29). When Levene's test for equality of variances is significant, Welch's t-test is used, which is an adaptation of Student's t-test (30), and is more reliable when the two samples have unequal variances and unequal sample sizes (31). An alpha level was set to 5\% with a significance level of $95 \%$, and a beta error accepted up to $20 \%$ with a power of study of $80 \%$.

\section{RESULTS}

\section{Histologic analysis}

Resorption of the disto-buccal root of the right maxillary first molar was found to be decreased in the laser group (L) (figure 2). Root resorption was the largest and deepest up to the dentin in the control group (C) (figure 1).
Odontoclasts were observed especially in the $\mathrm{C}$ group representing still active root resorption activities (Figure 3). Multiple dilated blood capillaries filled with blood cells were detected specially in the Lased group (L) even at compression side (Figure 2 and 4). Almost all of the root resorption craters of the $\mathrm{L}$ group showed deposition of newly formed reparative cementum covering the surface of resorption lacunae (Figure 4), while root resorption lacunae in $\mathrm{C}$ group showed less extension or even absence of the reparative cementum layer in most of the root resorption craters (Figure 1 and 3).

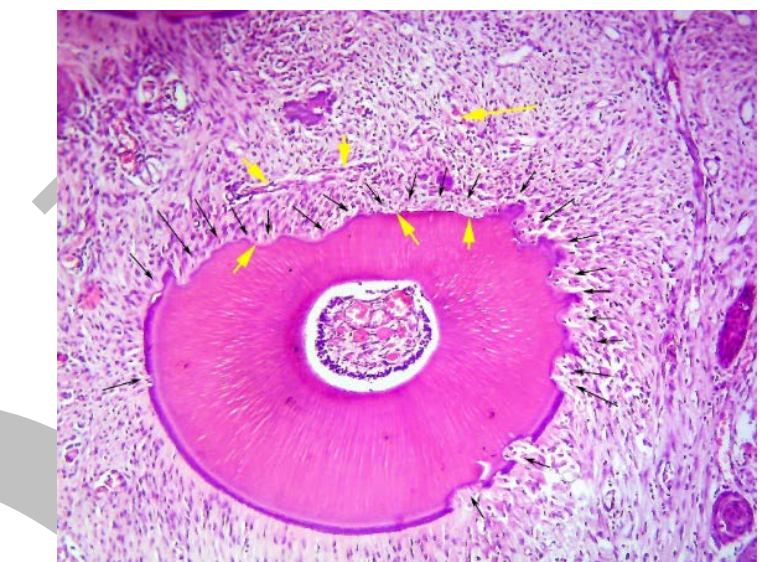

Figure (1): L.M. (C group) showing large area of root resorption (black arrows) with minimal reparative cementum and reduced number of blood capillaries at pressure side (yellow arrows) H\&E (100x)

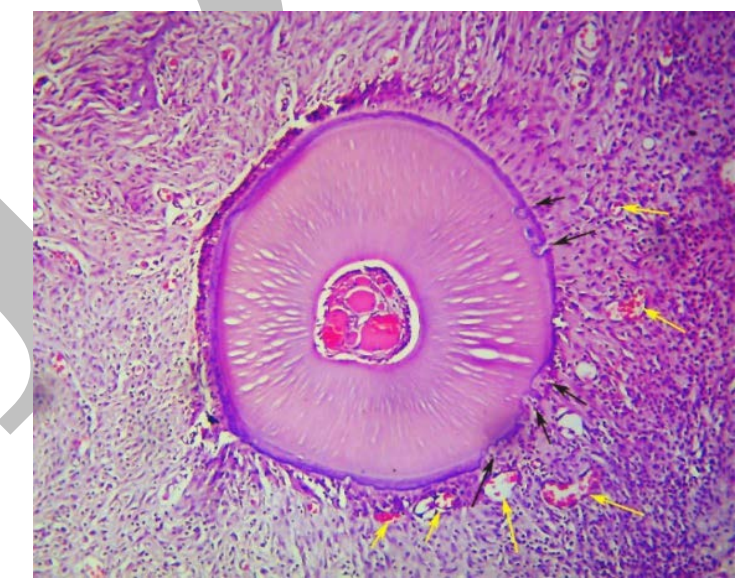

Figure (2): L.M. \{L group $\}$ showing small areas of root resorption (black arrows) with large number of blood capillaries found in the pressure side (yellow arrows). H\&E (400x)

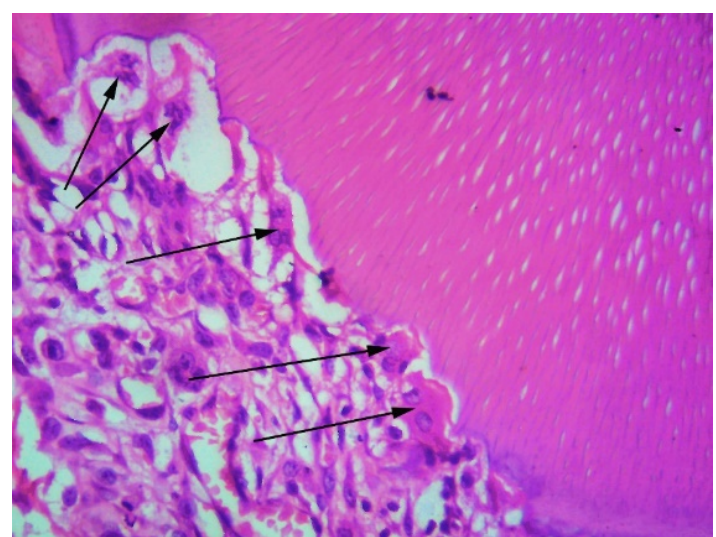

Figure (3): L.M. \{C group\} showing large area of root resorption, with odontoclasts (arrows) in their howships' lacunae and nearly absence of reparative cementum. H\&E (400x) 


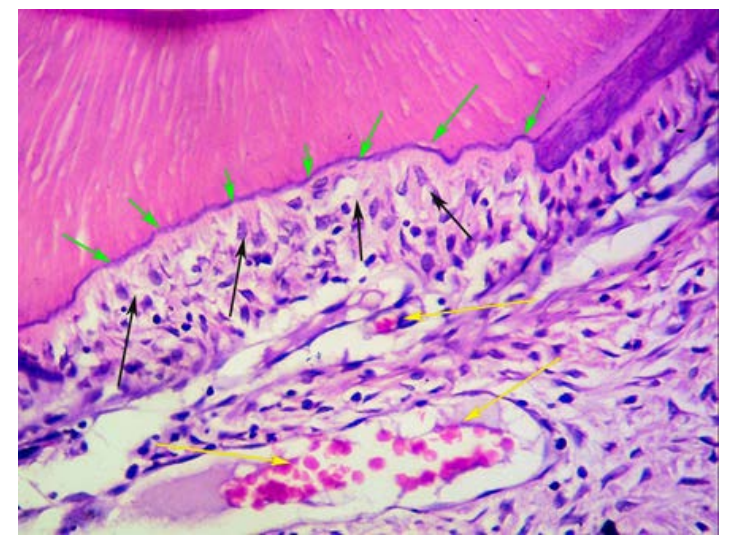

Figure (4): L.M. \{L group $\}$ showing area of root resorption (black arrows) covered with layer of reparative cementum (green arrows). Note the dilated blood capillaries in the compression side (yellow arrows). H\&E (400x)

\section{Histomorphometric analysis}

Root resorption was observed in both groups. The histomorphometric analysis of $\mathrm{H} \& \mathrm{E}$ stained slices showed statistically significant $(\mathrm{p}=0.001)$ reduction in amount of root resorption in the lased group in comparison to those of the control group (table1 and figure 5).

Table (1): Comparison between the two studied groups according to percentage of root resorption

\begin{tabular}{|l|c|c|c|}
\hline & $\begin{array}{c}\text { Control } \\
\text { high }\end{array}$ & Laser high & $\begin{array}{c}\text { Test of } \\
\text { significance } \\
\boldsymbol{p} \text { value }\end{array}$ \\
\hline Size of root & & & \\
$\quad$ resorption & 8 & 8 & \\
(\%) & $1.27-22.29$ & $0.41-3.74$ & $\mathrm{Z}_{(\mathrm{MW})}=4.468$ \\
-Min-Max & $9.57 \pm 6.14$ & $1.53 \pm 1.08$ & $p=0.001^{*}$ \\
-Mean \pm Std. & $5.669-$ & $0.847-$ & \\
$\quad$ Deviation & 13.474 & 2.2136 & \\
-95\% CI for mean & $8.07(6.27-$ & $1.10(0.76-$ & \\
-Median (IQR) & $14.19)$ & $2.06)$ & \\
-KS test of & $\mathrm{D}=0.211$, & $\mathrm{D}=0.204$, & \\
normality & $\mathrm{p}=0.146 \mathrm{NS}$ & $\mathrm{p}=0.178 \mathrm{NS}$ & \\
\hline
\end{tabular}

$\mathrm{n}$ : Number of rats, Min-Max: Minimum - Maximum, CI: Confidence interval, IQR: Inter-quartile range,

KS: Kolmogorov-Smirnov, MW: Mann-Whitney U test, *: Statistically significant $(\mathrm{p}<0.05)$

NS: Statistically not significant $(\mathrm{p}>0.05)$

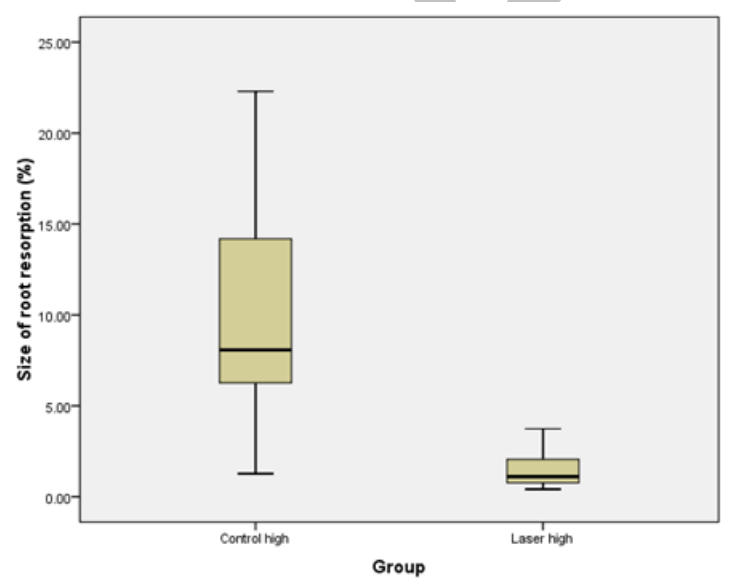

Figure (5): Box and whisker graph of size of root resorption (\%) in the studied groups, the thick line in the middle of the box represents the median, the box represents the inter-quartile range (from $25^{\text {th }}$ to $75^{\text {th }}$ percentiles), the whiskers represents the minimum and maximum.

\section{DISCUSSION}

Orthodontic tooth movement (OTM) is a process done by application of an active mechanical force that results in pressure and tension sites within the periodontal ligament. The periodontal ligament fibroblasts mediate pseudoinflammatory, multicellular, immunological process which provokes osteoclasts differentiation through the RANKL/OPG system and thus, osteoclastic bone resorption at pressure sites of the periodontal ligament, while bone formation by osteoblasts occurs in sites of tension (32). Root resorption is considered one of the unavoidable side effects of orthodontic treatment. Previous Studies showed the prevalence of OIIRR in orthodontic treated adolescents to be more than 90\%, approximately $15 \%$ exhibiting moderate to severe while $10-20 \%$ of the patients are manifested with severe resorption more than $3 \mathrm{~mm}(5,6)$.

Many chemicals/pharmacological agents (fluoride (12), steroidal and nonsteroidal drugs $(4,13)$, calcitonin (14), clodronate (16), Icariin (17), and tetracycline $(5,18)$ ) have been tested to prevent or to repair OIIRR. Although most of them were found effective, they are not clinically available due to their possible systemic or side effects on orthodontic tooth movement. (5) An alternative noninvasive and clinically suitable method to prevent and repair the OIIRR are low-level laser therapy $(\operatorname{LLLT})(1,4,5)$.

This study used induced orthodontic tooth movement in rats as a model to assess the effect of LLLT on orthodontic inflammatory root resorption. Previous studies using rat model to evaluate the OIIRR used amount of force varies from 10 to $100 \mathrm{~g}$ (11-13). We chose the coil spring of $50 \mathrm{~g}$ to induce mesial movement of the first maxillary molars and to ensure root resorption in the present study based on data of several previous studies (1, 5, 24). Since the human molar is almost 20 times larger than a rat molar, The coil springs of $50 \mathrm{~g}$ used in a rat should correspond to $1000 \mathrm{~g}$ in a human molar, which is considered as heavy force (33). However, we considered the relatively short experimental duration and aimed to cause significant resorption lacunae rather than smooth concavities in order to represent the possible early and late differences between groups.

There are many advantages of using rats as a model for orthodontic tooth movement. First, rats have periodontal tissue and anatomical structure close to those in humans. Second, rats are of low cost and easy to breed, making them compatible for a large sample study. Finally, orthodontic histological changes in the periodontal tissue and the mechanism are similar to those in humans, but change faster (14).

The average life expectancy of rats is approximately 2 years, whereas the worldwide average life expectancy in human population is approximately 70.5 years. Converting rat age to human age, a 21-day-long period for rats is equivalent to two years for humans, which is the typical length of time required for most of orthodontic treatment. This duration is also based on data of a previous study of Hikida et al. (34) in rat model. Moreover, previous study of Hellsing and Hammarstrom (35) reported definitive resorption sites forming within 1 week of orthodontic force application. Likewise, Jager et al. (36) showed the repair of root resorption craters started 9 days after appliance insertion. Hence, an experimental period of 21 days seems to be sufficient to produce resorption lacunae and start of root repair for investigation. 
In the present study, we reported a significant reduction in percentage of root resorption ( $\mathrm{p}=0.001)$ in the lased group (L) in comparison to that of the control group (C). This finding corresponds to the results of previous studies that reported the positive effect of LLLT in reduction of amount of root resorption after orthodontic tooth movement $(1,5$, $21,24)$. The mechanisms of reduction of OIIRR by LLLT were best described by Suzuki et al. (1) who suggested that the increase of osteoclastic activities and RANKL and TRAP expression at the compression sites after laser irradiation could lead to accelerated reduction of bone area at the compression sites, and consequently rapid reduction of the force at the tooth/ligament/bone interface. The rapid force reduction at periodontal ligament leads to less formation of hyalinized necrotic tissues, thus decreases the OIIRR as it is mainly formed due to elimination proses of the hyalinized tissues. In addition to that, laser biomodulation accelerates the inflammatory events and modulates the inflammation process to be lower and shorter, this provides more protection of the cementoblasts and cementoid layer as they will not subjected to a high level of stress for a longer duration. This could positively reduce the risk of root resorption and root damage even with the high bone resorption activity on the compression side.

In addition, and according to the light microscopy qualitative analysis, we observed increased in number of accentuated blood capillaries filled with blood cells in the $\mathrm{L}$ group greater than the $\mathrm{C}$ group. This observation corroborates with those of Akın et al. (37) and Yoo et al. (38) who reported that the absorption of Laser photons by mitochondria increases the levels of reactive oxygen species (ROS) and nitric oxide (NO). The NO could enhance the capillary vascularization and increase microvascular permeability. These findings, in addition to our results, support the hypothesis that LLLT may decrease the amount of OIIRR due to its effect on vasculature as the increased microvascular permeability could lead to increase in number of osteoclasts. In addition to that, the improvement of tissue oxygenation may activate cell signaling pathways, promoting transcription factors, such as nuclear factor kappa B (NF-kB), which is essential factor for RANKL induced osteoclast differentiation (39). The increase in osteoclastic bone resorption activities leads to rapid reduction of compressive forces that leads to less hyalinized tissue formation $(1,24)$. Furthermore, according to Brezniak and Wasserstein (3), the OIIRR is part of elimination of necrotic tissues formed due to cut of the blood supply to the periodontal tissues. So, we suggest that the enhancement of tissue vascularization may lead to less hyalinization and tissue necrosis from the start, so more protection of radical tissues.

Besides, our light microscopy qualitative analysis revealed an obvious formation of new layer of reparative cementum covers almost all the pre-formed resorption craters in the $\mathrm{L}$ group. In contrast, rats of $\mathrm{C}$ group showed less incidence and smaller thickness of newly formed reparative cementum. This inference is in accordance with the findings of Altan et al. (5) who reported the positive role of LLLT on acceleration and enhancement of healing and repair process of OIIRR. They claimed that LLLT accelerates the Initial secretion of uncalcified cementoid layer by repair cells, fibroblast-like cells and cementoblasts, followed by re-attachment of the periodontal ligament fibers (40). This provides rapid coverage of the radicular tissues, protecting them from the action of the resorptive cells. In addition, they suggested that LLLT accelerates and enhances the removal of the hyalinized tissue due to its antiinflammatory effects, in addition to acceleration and improvement of the reparative events of the OIIRR by controlling the RANKL/OPG ratio and the activities of the repair cells (5).

On the other hand, some studies reported disagreement in their results with our study (23). They reported that laser irradiation does not have a significant role in preventing and reducing the OIIRR. These conflicting results may be due to the use of insufficient force magnitudes or different laser parameters.

\section{CONCLUSION}

According to this study, amount of orthodontic induced inflammatory root resorption (OIIRR) was significantly reduced after low-level laser therapy (LLLT) with accelerated reparative events of the resorption craters. Therefore, we suggest that LLLT seems promising in preventing or reducing orthodontic root resorption, in addition to accelerating and enhancing the healing of OIIRR.

\section{CONFLICT OF INTEREST}

The authors declare that they have no conflicts of interest.

\section{REFERENCES}

1. Suzuki SS, Garcez AS, Suzuki H, Ervolino E, Moon W, Ribeiro MS. Low-level laser therapy stimulates bone metabolism and inhibits root resorption during tooth movement in a rodent model. J Biophotonics. 2016;9(1112):1222-35.

2. Shah A. Orthodontically Induced Root Resorption. IJARnD. 2017;2(1):18-25.

3. Brezniak N, Wasserstein A. Orthodontically induced inflammatory root resorption. Part I: The basic science aspects. Angle Orthod. 2002;72(2):175-9.

4. Fonseca PD, de Lima FM, Higashi DT, Koyama DF, Toginho Filho Dde O, Dias IF, et al. Effects of light emitting diode (LED) therapy at $940 \mathrm{~nm}$ on inflammatory root resorption in rats. Lasers Med Sci. 2013;28(1):49-55.

5. Altan AB, Bicakci AA, Mutaf HI, Ozkut M, Inan VS. The effects of low-level laser therapy on orthodontically induced root resorption. Lasers Med Sci. 2015;30(8):2067-76.

6. Weltman B, Vig KW, Fields HW, Shanker S, Kaizar EE. Root resorption associated with orthodontic tooth movement: a systematic review. Am J Orthod Dentofacial Orthop. 2010;137(4):462-76; discussion 12A.

7. Harris EF, Kineret SE, Tolley EA. A heritable component for external apical root resorption in patients treated orthodontically. Am J Orthod Dentofacial Orthop. 1997;111(3):301-9.

8. Nieto-Nieto N, Solano JE, Yanez-Vico R. External apical root resorption concurrent with orthodontic forces: the genetic influence. Acta Odontol Scand. 2017;75(4):280-7.

9. Fox N. Longer orthodontic treatment may result in greater external apical root resorption. Evid Based Dent. 2005;6(1):21-.

10. Nanekrungsan K, Patanaporn V, Janhom A, Korwanich N. External apical root resorption in maxillary incisors in orthodontic patients: associated factors and radiographic evaluation. Imaging Sci Dent. 2012;42(3):147-54. 
11. Gonzales C, Hotokezaka H, Yoshimatsu M, Yozgatian JH, Darendeliler MA, Yoshida N. Force magnitude and duration effects on amount of tooth movement and root resorption in the rat molar. Angle Orthod. 2008;78(3):502-9.

12. Gonzales C, Hotokezaka H, Karadeniz EI, Miyazaki T, Kobayashi E, Darendeliler MA, et al. Effects of fluoride intake on orthodontic tooth movement and orthodontically induced root resorption. Am J Orthod Dentofacial Orthop. 2011;139(2):196-205.

13. Gonzales C, Hotokezaka H, Matsuo K, Shibazaki T, Yozgatian JH, Darendeliler MA, et al. Effects of steroidal and nonsteroidal drugs on tooth movement and root resorption in the rat molar. Angle Orthod. 2009;79(4):715-26.

14. Guan L, Lin S, Yan W, Chen L, Wang X. Effects of calcitonin on orthodontic tooth movement and associated root resorption in rats. Acta Odontol Scand. 2017;75(8):595-602.

15. Hu Y, Liu W, Liu Z, Kuang W, He H. Receptor activator of nuclear factor-kappa ligand, OPG, and IGF-I expression during orthodontically induced inflammatory root resorption in the recombinant human growth hormonetreated rats. Angle Orthod. 2015;85(4):562-9.

16. Liu L, Igarashi K, Haruyama N, Saeki S, Shinoda H, Mitani H. Effects of local administration of clodronate on orthodontic tooth movement and root resorption in rats. The European Journal of Orthodontics. 2004;26(5):469-73.

17. Wang F, Liu Z, Lin S, Lu H, Xu J. Icariin enhances the healing of rapid palatal expansion induced root resorption in rats. Phytomedicine. 2012;19(11):1035-41.

18. Baysal A, Uysal T, Ozdamar S, Kurt B, Kurt G, Gunhan O. Comparisons of the effects of systemic administration of Lthyroxine and doxycycline on orthodontically induced root resorption in rats. Eur J Orthod. 2010;32(5):496-504.

19. Al-Daghreer S, Doschak M, Sloan AJ, Major PW, Heo G, Scurtescu C, et al. Effect of low-intensity pulsed ultrasound on orthodontically induced root resorption in beagle dogs. Ultrasound Med Biol. 2014;40(6):1187-96.

20. Seifi M, Atri F, Yazdani MM. Effects of low-level laser therapy on orthodontic tooth movement and root resorption after artificial socket preservation. Dent Res J (Isfahan). 2014;11(1):61-6.

21. Altan BA, Sokucu O, Ozkut MM, Inan S. Metrical and histological investigation of the effects of low-level laser therapy on orthodontic tooth movement. Lasers Med Sci. 2012;27(1):131-40.

22. Kim SJ, Chou MY, Park YG. Effect of low-level laser on the rate of tooth movement. Semin Orthod. 2015;21(3):210-8.

23. Vasconcelos EC, Henriques JF, Sousa MV, de Oliveira RC, Consolaro A, Pinzan A, et al. Low-Level Laser Action on Orthodontically Induced Root Resorption: Histological and Histomorphometric Evaluation. J Lasers Med Sci. 2016;7(3):146-51.

24. Suzuki SS, Garcez AS, Reese PO, Suzuki H, Ribeiro MS, Moon W. Effects of corticopuncture (CP) and low-level laser therapy (LLLT) on the rate of tooth movement and root resorption in rats using micro-CT evaluation. Lasers Med Sci. 2018;33(4):811-21.
25. Cook D. Cell Imaging Techniques: Methods and Protocols. Br J Biomed Sci. 2006;63(2):102.

26. Schneider CA, Rasband WS, Eliceiri KW. NIH Image to ImageJ: 25 years of image analysis. Nat Methods. 2012;9(7):671-5.

27. IBM Corp. IBM SPSS Statistics for Windows, Version 21.0. Armonk, NY: IBM Corp.; Released 2012.

28. Field A. Discovering Statistics Using IBM SPSS Statistics. 4th ed. London, California, New Delhi: SAGE Publications Ltd; 2013.

29. Box JF. Guinness, Gosset, Fisher, and small samples. Statistical science. 1987:45-52.

30. Welch BL. The generalization ofstudent's' problem when several different population variances are involved. Biometrika. 1947;34(1/2):28-35.

31. Ruxton GD. The unequal variance t-test is an underused alternative to Student's t-test and the Mann-Whitney U test. Behav Ecol. 2006;17(4):688-90.

32. Meikle MC. The tissue, cellular, and molecular regulation of orthodontic tooth movement: 100 years after Carl Sandstedt. Eur J Orthod. 2006;28(3):221-40.

33. Ren Y, Maltha JC, Kuijpers-Jagtman AM. The rat as a model for orthodontic tooth movement-a critical review and a proposed solution. Eur J Orthod. 2004;26(5):483-90.

34. Hikida T, Yamaguchi M, Shimizu M, Kikuta J, Yoshino T, Kasai K. Comparisons of orthodontic root resorption under heavy and jiggling reciprocating forces during experimental tooth movement in a rat model. Korean J Orthod. 2016;46(4):228-41.

35. Hellsing E, Hammarstrom L. The hyaline zone and associated root surface changes in experimental orthodontics in rats: a light and scanning electron microscope study. Eur J Orthod. 1996;18(1):11-8.

36. Jäger A, Kunert D, Friesen T, Zhang D, Lossdörfer S, Götz W. Cellular and extracellular factors in early root resorption repair in the rat. Eur J Orthod. 2008;30(4):336-45.

37. Akın E, Gurton AU, Ölmez H. Effects of nitric oxide in orthodontic tooth movement in rats. Am J Orthod Dentofacial Orthop. 2004;126(5):608-14.

38. Yoo SK, Warita H, Soma K. Duration of orthodontic force affecting initial response of nitric oxide synthase in rat periodontal ligaments. J Med Dent Sci. 2004;51(1):83-8.

39. Yu M, Qi X, Moreno JL, Farber DL, Keegan AD. NF- $\kappa$ B signaling participates in both RANKL-and IL-4-induced macrophage fusion: receptor cross talk leads to alterations in NF- $\mathrm{KB}$ pathways. J Immunol. 2011:1002628.

40. Brudvik P, Rygh P. The repair of orthodontic root resorption: an ultrastructural study. Eur J Orthod. 1995;17(3):189-98. 